\title{
Viabilidad económica y financiera de la ganadería caprina extensiva en San Luis Potosí, México
}

\author{
Octavio Tadeo Barrera Perales \\ Universidad Autónoma Chapingo, México \\ obarrera@ciestaam.edu.mx \\ Leticia Myriam Sagarnaga Villegas \\ Universidad Autónoma Chapingo, México \\ sagarnaga.myriam@gmail.com \\ José María Salas González \\ Universidad Autónoma Chapingo, México \\ jmsalasgonzalez@gmail.com

\section{Juan Antonio Leos Rodríguez \\ Universidad Autónoma Chapingo, México jleos@ciestaam.edu.mx}

\section{Rodolfo Santos Lavalle} \\ Universidad Autónoma Chapingo, México \\ rsantoslavalle@yahoo.com.mx
}

Cita sugerida: Barrera Perales, O. T.; Sagarnaga Villegas, L. M.; Salas González, J. M.; Leos Rodríguez, J. A. y Santos Lavalle, R. (2018).Viabilidad económica y financiera de la ganadería caprina extensiva en San Luis Potosí, México. Mundo Agrario, 19(40), e077. https://doi.org/10.24215/15155994e077

Recibido: 18 de septiembre de 2017 - Aceptado: 1 de enero de 2018 - Publicado: 26 de marzo de 2018 


\section{Viabilidad económica y financiera de la ganadería caprina extensiva en San Luis Potosí, México}

Economic and financial viability of extensive caprine livestock in San Luis Potosí, Mexico

Octavio Tadeo Barrera Perales

Universidad Autónoma Chapingo, México

obarrera@ciestaam.edu.mx

Leticia Myriam Sagarnaga Villegas

Universidad Autónoma Chapingo, México

sagarnaga.myriam@gmail.com

José Maria Salas González

Universidad Autónoma Chapingo, México

jmsalasgonzalez@gmail.com

Juan Antonio Leos Rodríguez

Universidad Autónoma Chapingo, México

jleos@ciestaam.edu.mx

Rodolfo Santos Lavalle

Universidad Autónoma Chapingo, México

rsantoslavalle@yahoo.com.mx

\section{Resumen:}

En México, la caprinocultura es una actividad importante dado que genera ingreso y alimento para familias ubicadas en zonas áridas con pocas alternativas. El objetivo fue determinar la viabilidad económica y financiera de la ganadería caprina extensiva e identificar los factores de permanencia de la actividad, en San Luis Potosí. Para recabar la información se utilizó la técnica de paneles de productores. La caprinocultura es rentable en el corto y mediano plazo debido al uso de mano de obra familiar y al bajo costo de alimentación en agostadero, no obstante, la permanencia en el largo plazo es incierta ya que el costo de los factores de producción no es cubierto.

Palabras ClaVE: Caprinocultura, Ingreso, Costos, Economía campesina, Agostadero, México.

\section{ABStraCt:}

In Mexico, goat rising is an important activity because it generates income and food for families located in arid areas with few alternatives. The objective was to determine the economic and financial viability of the extensive caprine livestock and to identify the factors of permanence of the activity, in San Luis Potosí. To obtain the information the technique of producer panels was used. Goat livestock is profitable in the short and medium term due to the use of family labor and the low cost of feeding in the rangeland, however, the permanence in the long term is uncertain since the cost of the factors of production is not covered.

KEYWORDS: Goat, Income, Costs, Economy peasant, Desert rangeland, Mexico.

\section{INTRODUCCIÓN}

Por sus hábitos de consumo, la cabra (Capra hircus) es capaz de aprovechar la vegetación característica de zonas áridas, lo cual la convierte en la principal especie ganadera que se adapta y produce en condiciones desérticas, de ella se obtienen productos como carne, leche y queso que son consumidos y comercializados por la población que habita en estas regiones, siendo así una fuerte importante de alimentos e ingresos para productores con recursos naturales marginales. 
En los países latinoamericanos, México ocupa el primer lugar en inventario de caprinos, seguido de Brasil. El rebaño caprino mexicano consta de aproximadamente 9 millones de cabezas, de acuerdo con el Servicio de Información Agroalimentaria y Pesquera (SIAP, 2015), el cual en los últimos 10 años presentó una tasa media de crecimiento anual (TMCA) negativa de $-0.77 \%$.

A nivel nacional, 250 mil familias de las zonas rurales viven de la ganadería caprina. De éstas, 17.500 se ubican en el estado de San Luis Potosí, principalmente en las zonas áridas y semiáridas del norte del estado donde habita el 36\% la población, que vive en precarias condiciones de vida, con lo cual el estado ocupa el sexto lugar nacional en pobreza y marginalidad (CONEVAL, 2017). De acuerdo con el SIAP (2015), San Luis Potosí tiene un inventario caprino de 615.673 cabezas, que lo ubica en quinto lugar de importancia a nivel nacional; se producen 2.625,1 toneladas de carne en canal, cuyo valor posiciona al estado en el séptimo lugar nacional (SIAP, 2015), y el valor de la producción anual caprina se estimó en 240 millones de pesos, representando el 5.9\% del total nacional (SIAP, 2015).

De acuerdo con lo anterior y a pesar de la disminución del inventario (-50\%), en los últimos 20 años, la caprinocultura es la actividad pecuaria más importante en la región árida de San Luis Potosí.

Debido a la importancia social y económica en el medio rural, se han realizado diversos estudios sobre esta actividad, la mayoría de interés zootécnico, enfocados al manejo epidemiológico (Suárez, Fondraz, Viñabal, Martínez y Salatin, 2013), manejo zootécnico en zonas áridas (Silvestre, Naim, Cueto y Gibbons, 2012; Hernández, Luna, Camacho, Franco y Hernández, 2014) y mejoramiento genético (Torres-Vázquez, Valencia-Posadas, Castillo-Juárez y Montaldo, 2010; Castillo-Rodríguez, Rivera-Sandoval, González-Reyna y Martinez-González, 2013; Pesántez y Hernández, 2014).

También, se han realizado algunos estudios socioeconómicos, como: estudios de mercado (Hernández et al., 2014), importancia de las cabras en el entorno rural (Cauderales, Milano y Granda, 2014; Guzmán, 2014; López Damián, Álvarez Parra y Zuluaga, 2012); e incluso se ha estudiado su rentabilidad y costos de producción (Cruz-Torres et al. 2011, UNAM 2012, Rebollar-Rebollar et al. 2012, Orona Castillo et al. 2013, Orona et al. 2014). Específicamente para San Luis Potosí, se han realizado dos trabajos relacionados con la rentabilidad de la ganadería caprina extensiva en la zona del Altiplano Potosino (Gómez, 2007; BarreraPerales, 2013) no obstante, en estas investigaciones no se realiza un análisis sobre su viabilidad económica y financiera y el análisis de costos se limita a costos desembolsados.

La mayoría de los estudios de análisis económicos de la ganadería extensiva, no consideran el costo de la alimentación en pastoreo, es decir no se incluye el costo del agostadero como fuente de alimento, lo que ocasiona que se sobreestime la rentabilidad de estos sistemas de producción. Al omitir el costo del forraje de los agostaderos, se incentiva al sobrepastoreo debido a que estos pastizales son de acceso abierto, incurriendo así en la problemática de los recursos comunes planteada por Hardin, (1968).

Los costos son una importante herramienta de planeación y control de las actividades de una empresa. La estimación de costos de producción permite generar información para la toma de decisiones en aspectos como la inversión en innovaciones tecnológicas y cambios en el proceso de producción que influyen en la rentabilidad económica y competitividad de la empresa (Parkin y Loria, 2010; Cruz-Torres, GarcíaHernández, Espinosa-Ortiz y Araque-Herrera, 2011; Rebollar-Rebollar et al., 2012).

La dificultad principal, que se presenta en la cuantificación de costos, es la falta de información de los productores, por lo que la aplicación de cuestionarios no es viable para la obtención de datos de costos e ingresos. Cabe mencionar, que no se encontraron estudios fidedignos de costos de producción que se hayan basado en una muestra probabilística, por lo que el uso de la técnica de paneles (Pearson \& Gotsch \& Sjaiful, 2004), surge como una opción para realizar este tipo de estudios.

Los paneles se realizan bajo la conducción de un experto con conocimiento y experiencia en la actividad. Los participantes (panelistas) son productores líderes de opinión, que cuentan con experiencia, quienes proporcionan información considerada como valores modales (tendencia central), no parámetros estimados econométricamente, de muestras estadísticamente válidas. 
Muy pocos estudios han utilizado la técnica de paneles de productores para estudiar los costos y la viabilidad económica de la caprinocultura (Sagarnaga-Villegas et al. 2014). Esta técnica ha sido usada en el análisis de costos de producción en diferentes cultivos y tipos de ganadería que permiten comparar el desempeño de una empresa con otras similares (Zavala, Salas, Leos y Sagarnaga, 2012; Sagarnaga-Villegas et al., 2014; Vargas-Canales, Palacios-Rangel, Acevedo-Peralta y Leos-Rodríguez, 2015).

Si bien, la cuantificación de costos permite generar estrategias de mejora en los procesos técnicos y económicos de las unidades de producción, en la mayoría de los casos, éstos se analizan bajo un enfoque financiero, bajo el cual, al estimar indicadores económicos como la relación beneficio/costo y la rentabilidad, se esperarían resultados positivos para que la empresa obtenga ganancias y se considere viable; cuando estos parámetros son bajos o negativos, se consideraría que la empresa no está generando utilidades e inclusive está perdiendo dinero; por lo que, no se justificaría la continuidad de la misma.

No obstante, se pueden realizar otro tipo de análisis económicos para unidades de producción campesinas, que aunque presenten indicadores de rentabilidad negativos, siguen operando, ya que lo hacen bajo una lógica de reproducción diferente, tal como lo dice concretamente Landini, (2011) "cuando los campesinos toman decisiones relacionadas a la producción, comercialización, ahorro, inversión y consumo es decir, económicas lo hacen a partir de un conjunto de parámetros, reglas y supuestos propios, que no se identifican con la lógica capitalista de mercado" (p.2), de ahí la importancia de realizar un análisis de costos y rentabilidad económica que considere un enfoque de economía campesina retomando conceptos como el "excedente de fondo de reemplazo" propuesto por Wolf, (1971) que ayuden a comprender la lógica de este tipo de productores tal como lo muestran los estudios de Bartra, 1974 y Schejtman, 1981.

El objetivo de este trabajo fue determinar la viabilidad financiera y económica de la ganadería caprina extensiva, en el estado de San Luis Potosí, mediante la estimación de costos e ingresos financieros y económicos; así como identificar los factores que determinan la permanencia de esta actividad. La hipótesis que esta investigación plantea es que la ganadería caprina es una actividad aparentemente eficiente, en términos financieros, debido al bajo costo de la alimentación (pastoreo en el agostadero), que es su insumo principal; sin embargo, en términos económicos en realidad se está haciendo una extracción de recursos no sostenible, que agotan el agostadero.

\section{Metodología}

El estudio se realizó en los meses de junio de 2015 y 2016 en la región del Altiplano Potosino (Figura 1), una de las cuatro regiones biogeográficas de San Luis Potosí (coordenadas geográficas $24^{\circ} 29^{\prime} 27^{\prime \prime}$ a $21^{\circ} 09^{\prime}$ 33" latitud norte y $98^{\circ} 19^{\prime} 48^{\prime \prime}$ a $102^{\circ} 18^{\prime} 10^{\prime \prime}$ longitud oeste), caracterizada por ser una zona árida con una precipitación menor a los $400 \mathrm{~mm}$ anuales, suelos calcáreos y periodos de sequía y heladas (MirandaAragón, Treviño-Garza, González-Tagle, Pompa-Garcia y Aguirre-Salado, 2011), en esta región la ganadería -conformada principalmente por ganado caprino- es una de las principales actividades económicas. 
FIGURA 1

Mapa de la zona de estudio donde se ubican las URP en la región del

Altiplano Potosino. Elaboración: Paula Nephtaly Carrera Álvarez

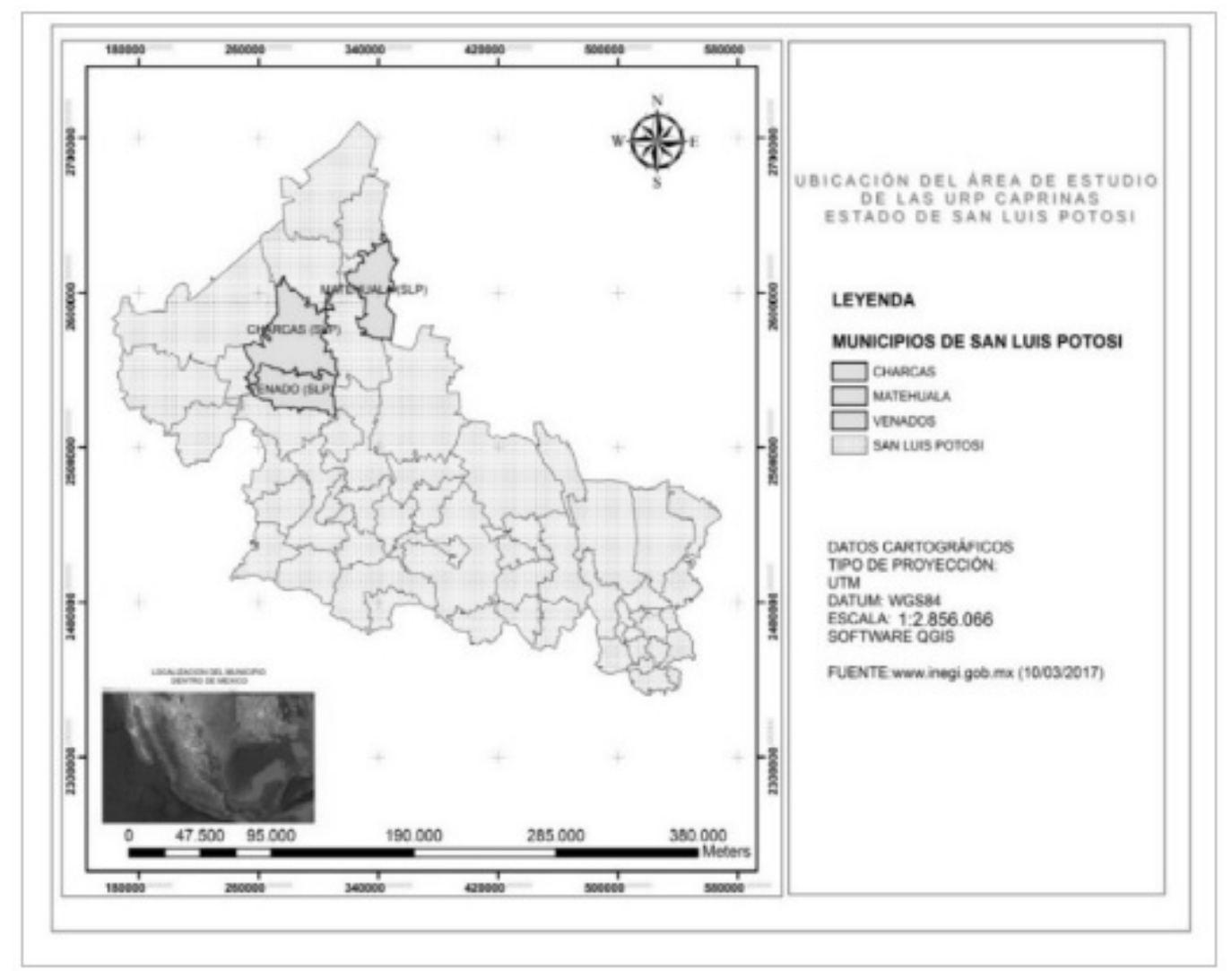

Para recabar la información requerida en el análisis, se utilizó la técnica de paneles de productores. Esta técnica fue desarrollada por el Centro de Política Agrícola y de los Alimentos (AFPC) de la Universidad de Texas A\&M. La cual fue adaptada para cuantificar ingresos y costos de producción de productos agrícolas en México por Salas, Sagarnaga, Gómez, Leos y Peña, (2013).

Los paneles de productores son una adaptación de la técnica Delphi, la cual es un método de investigación prospectivo cuyo objetivo fundamental es obtener una respuesta grupal fidedigna ( $y$ en la medida de lo posible consensuada) a partir de un proceso sistemático de recopilación y análisis de información (DomínguezTorreiro y Gómez-Rodríguez, 2013). De acuerdo a Sagarnaga-Villegas et al., (2014): "Un panel tiene el objetivo de construir una Unidad Representativa de Producción (URP), la cual es una explotación típica de una unidad de producción de una escala y un sistema de producción particular de una región”. El panel está formado por productores con características de producción similares (escala, cultivo o especie de ganado y parámetros técnicos), en este grupo se discute y consensa información sobre el manejo técnico, costos de insumos, rendimientos y precio de venta de un producto agrícola o ganadero (Sagarnaga-Villegas, SalasGonzález y Aguilar-Ávila 2014).

Con apoyo de la facilitadora estatal del Sistema Producto Caprino de San Luis Potosí, se identificaron las URP caprinas del estado, las que se denominaron SLCC80 y SLCC100. La nomenclatura de las URP se conformó de la siguiente forma; SL se refiere a San Luis Potosí; CC a caprinos carne y el número corresponde a la cantidad de vientres en producción. En el caso de SLCC80, el panel se realizó en junio de 2015 con la participación de siete productores del municipio de Matehuala. Para SLCC100, el panel se realizó en junio de 2016, con la participación de nueve productores de los municipios de Charcas y Venado. Los paneles se realizaron en las oficinas de la Secretaría de Desarrollo Agropecuario de San Luis Potosí (SEDAGRO). 
La selección de los panelistas se realizó a partir de un padrón de productores asociados al Sistema Producto Caprino de San Luis Potosí, mediante un muestreo no probabilístico de selección experta; este método se emplea para seleccionar unidades representativas de acuerdo a los criterios de un experto (Pimienta, 2000). Los criterios aplicados para seleccionar a los panelistas fueron el tamaño del rebaño (escala), nivel tecnológico, conocimiento e información de costos e ingresos y experiencia en la ganadería caprina, es decir, los panelistas que se consideraron para este trabajo son los que representan las características más comunes de los caprinocultores de la región de estudio, lo cual fue validado por los mismos productores y el facilitador experto.

La información que se obtuvo en los paneles consistió en parámetros zootécnicos, precios de insumos, factores de producción y precios. Esta información se sistematizó y proceso en Excel $^{\circledR}$ para construir presupuestos de las URP. Los resultados fueron validados posteriormente por los productores panelistas en una reunión planeada con este fin, para comprobar que reflejaran la situación técnica, económica y financiera de las URP analizadas.

La estimación de ingreso, costos, viabilidad financiera, viabilidad económica y relación beneficio/costo se realizó aplicando la metodología de la Asociación Americana de Economía Agrícola (United States Department of Agriculture - Natural Resources Conservation Service [USDA-NRCS], 2000), la cual fue ajustada para adecuarla al análisis de costos en el sector agropecuario de México.

Los ingresos y costos totales, así como la relación B/C se estimaron de la siguiente manera.

Ingreso total (IT):

$$
\begin{aligned}
& \text { IT = QiPi + IT + IO } \\
& \text { donde; } \\
& \text { I = Precio por cantidad de producto vendido } \\
& \text { IT= Ingreso por transferencias } \\
& \text { IO = Otros ingresos } \\
& \text { Donde Pi es el precio del producto } \\
& \text { Costos variables (CV): }
\end{aligned}
$$

Costos variables $(\mathrm{CV})$ : 


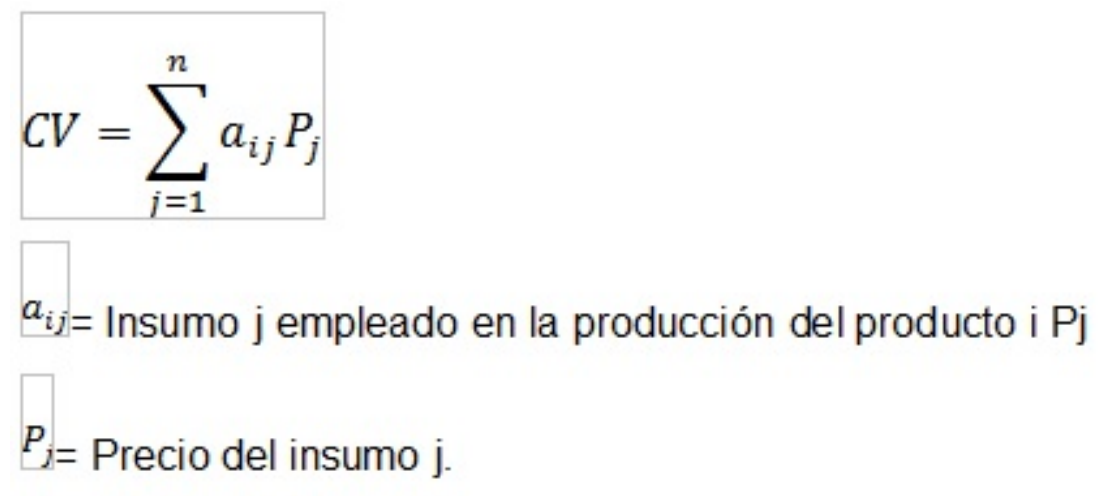

Costos fijos (CF):

$C V=\sum_{k=1}^{n} a_{i k} P_{k}$

donde:

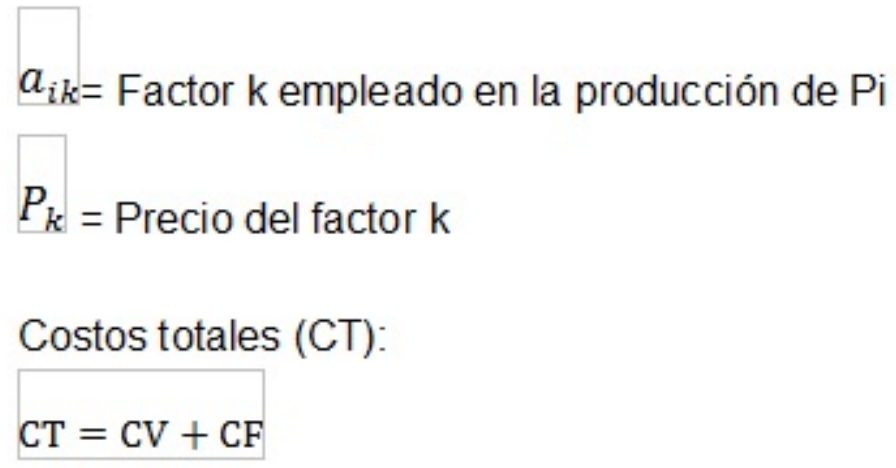

Relación beneficio/costo $(\mathrm{B} / \mathrm{C})$ :

$$
B / C=\frac{I T}{C T}
$$

Las variables estimadas fueron: Costos Fijos (CF), Costos Variables (CV) y Costos Totales (CT) desembolsados y no desembolsados, tanto en términos financieros, como económicos. A partir de las cuales se estimaron tres tipos de costos: a) Costos Financieros (CFIN), b) Costos desembolsados (CD) y c) Costos Económicos (CEC), lo que finalmente permitió determinar la viabilidad económica y financiera, así como el flujo de efectivo de las URP analizadas.

Finalmente, mediante un análisis de precios ponderados se identificó el número de vientres necesarios que se requieren para cubrir los costos desembolsados, financieros y económicos.

Los Costos Financieros (CFIN) son la sumatoria de los CF y CV desembolsados y no desembolsados, no incluyen los costos de oportunidad de los recursos propios, mano de obra familiar y gastos de gestión empresarial (United States Department of Agriculture-Economic Research Service, 2012). 
Los Costos Económicos (CEC) están compuestos por los CF y CV más el costo de oportunidad de los factores de producción, es decir, los costos de la tierra (que incluyen áreas de pastoreo), mano de obra propia y familiar no remunerada, capital neto de trabajo y capital invertido en infraestructura y semovientes (USDA -ERS, 2012). El Flujo Neto de Efectivo (FNE) es la sumatoria real en términos monetarios que una empresa recibe o paga por gastos de operación durante un periodo específico (Besley et al. 2008). El concepto de CEC se interpreta como el "costo de oportunidad" de los factores empleados en la actividad productiva (Parkin y Loria, 2010). Para estimarlo se consideraron los gastos en efectivo, incluyendo los retiros personales, y se excluyeron las amortizaciones.

La rentabilidad financiera y económica se consideró como la relación entre los beneficios generados por la actividad respecto a los gastos que fueron empleados para llevarla a cabo (García, 1999), su valoración se realizó mediante el cálculo de la relación beneficio costo $(\mathrm{B} / \mathrm{C})$ tanto en términos financieros como económicos. Los costos e ingresos se estimaron por separado y se consideró un año de operación en ambas URP; el año analizado se considera dentro del periodo de estabilidad económica del proyecto.

Los Costos Desembolsados (CD) son los costos efectivamente desembolsados por la empresa en el proceso de producción (Besley y Bringham, 2008), en su cálculo se consideran únicamente los CV y CF desembolsados, el pago de intereses a créditos de corto plazo y el pago a capital e intereses de créditos de largo plazo, en caso de existir; se excluyen las amortizaciones de activos fijos y semovientes (USDA -ERS, 2012), así como los costos de oportunidad de los factores de producción (capital, trabajo familiar y gestión empresarial).

Esta última opción es usada con relativa frecuencia en el análisis de la ganadería. (Santarcángelo y Fal, 2009; Cruz-Torres et al., 2011; Rebollar-Rebollar et al., 2012). Sin embargo, este tipo de análisis no es el más acertado para evaluar la viabilidad de una empresa; ya que sólo toman en cuenta los ingresos desembolsados de las explotaciones, menos los costos desembolsados que se generan en el proceso productivo; si bien indican la liquidez de la unidad de producción, que muestra la capacidad de esta para cumplir con obligaciones de corto plazo, no proporcionan una referencia para concluir sobre la continuidad, de mediano y largo plazo de la empresa, en función de la recuperación de sus factores de producción.

El análisis de precios compuestos se realizó mediante la ponderación de los ingresos de cada producto (queso, cabrito y desechos) con respecto al ingreso total obtenido en las URP, este precio se ponderó con el precio de venta y la cantidad generada de cada producto en ambas URP, así al dividir este dato entre el tamaño del rebaño y realizar la sumatoria de estos precios se estimó un precio ponderado compuesto por vientre. Lo que permitió determinar el número de vientres requeridos para cubrir los $\mathrm{CD}$, los CFIN y los CEC por cabra.

Cabe destacar que el análisis de costos y rentabilidad realizado en este trabajo se apoyó en un enfoque de economía campesina; ya que como lo menciona Vargas-Canales et al. (2015) el valor de la producción agropecuaria no siempre se basa en el dinero. Hay insumos como el pasto, agua, abono y mano de obra (familiar) que no se compran ni se venden; además, el autoconsumo de cosechas y ganado que la unidad familiar (campesina) realiza es un beneficio propio.

Para la estimación de indicadores de rentabilidad, ingresos y costos de producción, se consideró el tipo de cambio promedio a junio de 2016, de 18,7 pesos por dólar americano (BANXICO, 2017).

\section{RESULTADOS Y DISCUSIóN}

\section{Características de las URP analizadas}

El sistema de producción de ambas URP es extensivo, enfocado en la producción de cabrito y queso fresco artesanal; cuentan con instalaciones rústicas y la fuente principal de alimentación es el agostadero comunal con suplementación temporal, que es la práctica más común en zonas áridas; se practica el autoreemplazo de vientres, y se emplean dos trabajadores permanentes (mano de obra familiar). La duración de la lactancia es 
de 150 días, con una producción promedio de un litro, es decir; 150 litros de leche por cabra por lactancia, que procesada en queso da un rendimiento de 21.4 kilogramos de queso. El cabrito y el queso son vendidos en mercados locales y en la URP. El cabrito se vende de 15 a 30 días a partir de su nacimiento y se clasifica de acuerdo a su calidad en: cabrito de primera y cabrito supremo. Se producen 72 y 110 cabritos al año en SLCC80 y SLCC100 respectivamente (Tabla 1). Parte de los animales de desecho son destinados para el autoconsumo. Ambas URP son tomadoras de precios. Cabe señalar, que a pesar de la similitud técnica de las dos URP, se encontraron resultados diferentes que justifican su análisis. En San Luis Potosí, la Campaña Nacional Contra la Brucelosis en los animales reporta en CONTROL esta enfermedad (prevalencia mayor del 3\%); por lo que, no es una limitante comercializar queso y cabrito (SENASICA, 2017).

TABLA 1

Parámetros técnicos de las URP analizadas en San Luis Potosí

\begin{tabular}{|l|l|l|l|l|l|}
\hline URP & Vientres en producción & Fertilidad (\%) & Remplazo (\%) & Mortalidad (\%) & I/cabra/año*-1 \\
\hline SLCC80 & 80 & 70 & 7 & 10 & 150 \\
\hline SLCC100 & 100 & 80 & 10 & 10 & 150 \\
\hline
\end{tabular}

En ambas URP, la mano de obra empleada es de tipo familiar (productor y su esposa y/o hijos). Sobre este tema Shanín (1976, p.12) y Orozco Hernández y López Andrés, (2007) consideran que en la economía campesina, la explotación agropecuaria se basa en el trabajo familiar, siendo su actividad primordial el cultivo de la tierra y la cría del ganado con niveles de especialización limitada.

Las URP analizadas se enfocan en la cría de ganado caprino con parámetros zootécnicos bajos, ambas URP reportan en promedio $75 \%$ de fertilidad en vientres. Esta fertilidad se atribuye al bajo peso de los vientres al momento del empadre, ocasionado por falta de alimento y nulo manejo reproductivo, de acuerdo con Mellado (2008), el parámetro estimado está ligado a las condiciones del agostadero y disponibilidad del forraje, aunque es posible llegar a $80 \%$ de fertilidad con estrategias de alimentación y manejo. Sin embargo, Orona Castillo et al. (2013), para este tipo de sistemas, considera posible una fertilidad hasta del $90 \%$ cuando existen las condiciones adecuadas de alimentación.

La duración de la lactancia (150 días) y la producción de leche de en la misma (1 l/día), en ambas URP, se deben a la escasez de forraje y a los trayectos largos para el pastoreo, así como al bajo potencial genético del ganado para la producción de leche (Mellado, 2008; Rebollar-Rebollar et al., 2012; Barrera-Perales; 2013; Orona Castillo et al., 2013).

Con relación a la producción que se genera en las explotaciones campesinas, casi en su totalidad se destina para el autoconsumo, sin embargo, cuando existe excedente en la producción como en el caso de las URP analizadas, este es comercializado. En este sentido, Schejtman, (1995) y Santacoloma- Varón, (2015) comentan que las unidades de producción campesinas se relacionan con otros sistemas económicos a través del mercado. Como en este estudio, donde las URP comercializan el cabrito y el queso en mercados regionales y a intermediarios, por lo que este tipo de sistemas de producción extensivos son de suma importancia ya que son la base productora de alimentos para mercados especializados como el sector restaurantero a donde se dirige la producción de la caprinocultura del Altiplano Potosino.

El sistema de producción caprino extensivo permite obtener ingresos y acceso al autoconsumo de proteína (leche y carne) en zonas áridas; algunas características como la mano de obra familiar y la articulación con mercados locales son similitudes que se observan en otras regiones áridas de México como en el Bajío e incluso en otros países como Benín (Dossa, Rischkowsky, Birner, \& Wollny, 2008) y Costa de Marfil (Duflo \& Udry, 2003) en el continente africano. En américa latina este sistema es similar en Perú y Bolivia, observándose 
en común los recursos naturales de las zonas áridas y el deterioro ambiental de las mismas a causa del sobrepastoreo y la tenencia de la tierra (Coppock et al., 2017).

\section{INGRESOS TOTALES}

Los ingresos de SLCC 80 y SLCC100 provienen de tres fuentes: queso fresco artesanal (tipo panela), cabrito para abasto y animales de desecho. Los panelistas dijeron no recibir transferencias gubernamentales, por lo que no fueron consideradas.

Cabe destacar que la leche no se comercializa en fresco, ésta es empleada para elaborar queso; se requieren 7 litros para obtener un kilogramo de queso, el cual se comercializa en piezas de $250 \mathrm{~g}$. El precio de venta del cabrito puede fluctuar en más menos 30\%; esta variación es ocasionada por el incremento en la demanda que se observa en diferentes épocas del año (fin de año y festividades) y la estación del año (lluvia o sequía). Para este trabajo se consideró un precio de $\$ 25,4$ USD por cabrito y $\$ 4$ USD por kg de queso para ambas URP (Tabla 2).

TABLA 2

Producción e ingresos en las URP analizadas en San Luis Potosí

\begin{tabular}{|l|l|l|l|l|l|l|}
\hline & \multicolumn{3}{|c|}{ SLCC80 } & \multicolumn{3}{c|}{ SLCC100 } \\
\hline Productos & Cantidad & Ingresos ${ }^{\wedge}$ & $(\%)$ & Cantidad & Ingresos $^{\wedge}$ & $(\%)$ \\
\hline Queso & $916^{\star}$ & $3.674,4$ & 60 & $1.143^{\star}$ & $4.585,9$ & 57 \\
\hline Cabrito & $72^{\star \star}$ & $1.828,9$ & 30 & $110^{\star *}$ & $2.794,1$ & 35 \\
\hline Desechos & $487^{\star * \star}$ & 580,2 & 10 & $413,2^{\star \star *}$ & 640,8 & 8 \\
\hline Ingreso total & & $6.083,5$ & 100 & & $8.020,9$ & 100 \\
\hline
\end{tabular}

En esta investigación se tenía la idea preconcebida de que el principal ingreso de las URP se percibiría por la venta de cabrito, sin embargo, al analizar la información se encontró que el ingreso principal es obtenido por la venta de queso. Lo anterior concuerda con Barrera-Perales (2013), quien reportó que para explotaciones caprinas extensivas en el norte de San Luis Potosí, la venta de queso fue la principal fuente de ingresos (36\% del ingreso total), mientras que el cabrito representó el $24 \%$ de sus ingresos totales. De manera similar, en Venezuela, las explotaciones caprinas bajo pastoreo extensivo obtienen $81 \%$ del total de ingresos de la venta de leche y $14 \%$ de la venta de cabritos (Cruz-Torres et al., 2011).

Los panelistas de ambas URP consideraron como principal fuente de ingreso a los cabritos; esto se debe a que los ingresos que se obtienen por la venta de dichos animales, se reciben acumulados en dos periodos (marzo-abril y agosto-septiembre); mientras que el ingreso percibido por la venta de queso, a pesar de que es mayor, se distribuye en pequeñas cantidades a lo largo del año.

Una práctica común es que en la época de estiaje (marzo - junio), para evitar pérdidas económicas, por la muerte de animales debido a la falta de alimento, los productores se ven obligados a vender los cabritos al mejor postor, a un precio que no consideran justo. 


\section{Costos FinANCIERos}

De manera agregada, en términos financieros, los CV representan 72\% para SLCC80 y $56 \%$ para SLCC100; y los CF $28 \%$ para SLCC 80 y 44\% para SLCC100. Lo cual difiere de lo encontrado en otros trabajos, en los cuales se reportan CV que van de 43\% a 52\% y CF que van de 43\% a 56\% (Cruz-Torres et al., 2011; RebollarRebollar et al., 2012). Lo anterior se debe a que las URP analizadas en este trabajo cuentan con infraestructura rústica y escaso o nulo equipamiento, lo que se refleja en bajos costos por concepto de depreciación.

Es bien sabido que la alimentación es el concepto de mayor peso en la producción ganadera, llegando a representar hasta el $80 \%$ de los costos totales de producción que se desembolsan en las explotaciones pecuarias (FAO, 2017).

En este análisis se encontró que el costo de alimentación es bajo en ambas URP, para SLCC80 representa el 4\% y para SLCC100 el 15\%, esto es debido a que la principal fuente de alimento es el agostadero y sólo se incurre en el costo de sales minerales para suplementación en época de estiaje y mano de obra de un pastor para su cuidado. De manera semejante, en Venezuela, una explotación caprina bajo un sistema de producción similar tiene un costo de alimentación del 6\% al 12\% (Cruz-Torres et al., 2011). No obstante, hay otros trabajos en México, en los cuales para sistemas de producción en pastoreo no se considera el costo de alimentación, esto ocasiona que se perciban altos márgenes de utilidad (Rebollar-Rebollar et al., 2012).

El costo de producción estimado por vientre es muy similar para ambas URP, y en SLCC80 va de \$12,7 USD en término de costos desembolsados, a \$85,5 USD en términos económicos, y para SLCC100, este costo va de $\$ 11,7$ USD en costos desembolsados a \$86,5 USD en términos económicos (Tabla 3.).

TABLA 3

Costos por vientre y totales de las URP analizadas en San Luis Potosí

\begin{tabular}{|l|c|c|c|}
\hline URP & CD (USD) & CFIN(USD) & CEC(USD) \\
\hline SLCC80 (vientre) & 12,7 & 17,7 & 85,5 \\
\hline SLCC80 (URP) & $1.014,9$ & $1.413,1$ & $6.838,7$ \\
\hline SLCC100 (vientre) & 11,7 & 20,4 & 86,5 \\
\hline SLCC100 (URP) & $1.166,9$ & $2.043,4$ & $8.651,2$ \\
\hline
\end{tabular}

\section{Costos DESEMBoLSADOS}

En el análisis de CD no se consideraron abonos a capital, ni pago de intereses, debido a que los productores no cuentan con créditos; se observa que los costos desembolsados por vientre son mayores en SLCC80, esto obedece a las economías de escala dónde entre más pequeña sea una unidad de producción estos costos tienden a incrementarse.

Costos Económicos

En los costos económicos, se incluyó el costo de oportunidad de los factores de producción. Para el costo de la tierra, los panelistas consideraron que los terrenos de agostadero no tienen costo de oportunidad, ya que no son adecuados para la producción agrícola por lo que no tienen valor. En el estado de Hidalgo, Sagarnaga et al. (2015) reportó que para los ganaderos, los agostaderos se consideran como "terrenos pobres, no aptos para cultivos agrícolas, para los que no hay un mercado desarrollado". De manera similar, en Argentina, la ganadería extensiva se desarrolló en áreas ubicadas en zonas áridas por su nula vocación agrícola y el bajo precio de las grandes extensiones para pastoreo (Ensabella, 2008). 
No obstante, los panelistas estimaron el costo de oportunidad del agostadero, partiendo del supuesto de la renta de una superficie de 30 hectáreas para pastoreo, que asciende a $\$ 802,1$ USD anuales para el caso de SLCC80 y para SLCC100 la renta de 45 ha de agostadero se estimó en \$1.336,9 USD.

El costo de oportunidad del agostadero también puede ser estimado al comparar el costo que tendría alimentar a los rebaños de forma estabulada. Una dieta que cubra los requerimientos nutricionales de las cabras tiene un costo de $\$ 0,3 \mathrm{USD} / \mathrm{kg}$, si se considera un suministro de $0.3 \mathrm{~kg}$ por día por animal en producción (Torres Rivera et al., 2013). De esta manera se estima un costo de oportunidad del agostadero de $\$ 1,975,8$ USD para SLCC80 y de \$2,135,5 USD para SLCC100.

En Estados Unidos de Norteamérica se han explorado otras formas de valorar el forraje de los agostaderos a través de diversos métodos como la valoración contingente (Hof, McKean, Taylor, \& Bartlett, 1989), cuotas de pastoreo, comparación de mercado y costo total de aprovechamiento (Bartlett, Torell, Rimbey, Tassell, \& Daniel, 2002).

$\mathrm{Al}$ incluir el costo del agostadero, estimado por cualquiera de los dos primeros métodos mencionados, las URP dejan de ser viables en términos financieros e incluso las necesidades de efectivo son superiores al efectivo generado, por lo que dejan de tener liquidez.

Es decir, la alimentación en agostadero permite que la ganadería caprina sea rentable; de otro modo, se tendría que desembolsar una cantidad importante de dinero para la compra de insumos para la alimentación en corral. En lo anterior coinciden varios autores, quienes afirman que la viabilidad de este tipo de URP se debe principalmente al bajo costo de alimentación basado en pastoreo (Cruz-Torres et al., 2011; Orona et al., 2014; Rebollar-Rebollar et al., 2012).

Para México, la Comisión Técnico Consultiva de Coeficientes de Agostadero (COTECOCA) mide la capacidad de los agostaderos para mantener a las especies ganaderas mediante el uso de un coeficiente denominado Unidad Animal (UA) Una UA corresponde a una vaca de $450 \mathrm{~kg}$ con su cría; una cabra representa solamente 0.17 de una UA. El Instituto Nacional de Investigaciones Forestales Agrícolas y Pecuarias (INIFAP), estimó un coeficiente de agostadero de 17 ha/UA/año-1 (INIFAP, 2008) para la región del Altiplano Potosino, lo que indica que 17 hectáreas de agostadero producen el forraje requerido para mantener hasta 6 cabras productivas. Los productores participantes en este análisis mantienen en los agostaderos un número de cabras mucho mayor. Es decir, los productores hacen un uso ineficiente del agostadero lo cual repercute en su sostenibilidad, poniendo en riesgo el futuro potencial de este recurso natural.

$\mathrm{Al}$ respecto Ensabella, (2008) sostiene que hay evidencia de que el deterioro de los recursos naturales, en particular aquellos de las zonas áridas, ocasionan migración de la población y pobreza por la pérdida de la productividad de estos ecosistemas. De acuerdo a la Comisión Económica para América Latina y el Caribe (CEPAL); países como México, Paraguay y Argentina presentan un deterioro ocasionado por la desertificación de sus zonas áridas en más de la mitad de su territorio, atribuido principalmente al sobrepastoreo y deforestación (CEPAL, 2005), es por ello que se debe poner un especial énfasis en el costo económico de los agostaderos de las zonas áridas.

El costo de oportunidad de la mano de obra del productor y su familia, fue estimado en dos jornales diarios, los cuales se cotizaron igual que un jornal en la zona. Es importante señalar, que de no ser por la ganadería caprina, sería difícil que las personas se empleen la mayor parte del año en otras actividades económicas, especialmente cuando son adultos mayores o niños. El costo de oportunidad de las actividades gerenciales (encargado de la URP) se estimó a partir del salario de un empleado especializado, que podría desarrollar esta actividad con un pago promedio de $\$ 65,2$ USD mensuales.

En San Luis Potosí en 2016, el sueldo promedio (\$11,2 USD) estuvo compuesto por tres salarios mínimos ( $\$ 3,7$ USD), y un jornal en la región del altiplano tuvo un costo promedio de \$7,5 USD (Comisión Nacional de Salarios Mínimos, 2016), es decir, la ganadería caprina de acuerdo a los ingresos estimados en ambas URP, podría cubrir en el caso de SLCC80 un salario conformado por \$3,5 USD, es decir, poco menos de la mitad 
de un jornal, mientras que para SLCC100 se podría cubrir un salario de \$4,4 USD. Lo anterior pone en evidencia la importancia de la actividad para generar ingreso, si bien modesto, en zonas donde no hay otras posibilidades de empleo o autoempleo.

$\mathrm{Al}$ respecto, bajo un enfoque de economía campesina la mano de obra familiar empleada en la producción se convierte en un ingreso para el productor, como lo menciona Bartra (1976) ya que "el producto obtenido por el trabajo familiar es la única categoría posible de ingreso en ausencia de salarios" por la escasa demanda; por eso es que la ganadería resulta una actividad de vital importancia en la generación de empleo en las zonas rurales.

El costo de oportunidad del capital de trabajo (capital necesario para cubrir un mes de operaciones de las URP), más el costo del capital invertido en semovientes, instalaciones, maquinaria y equipo, se estimó a partir de una tasa de interés de $8 \%$ (costo de oportunidad del capital invertido en activos fijos y capital neto de trabajo), que es el costo de oportunidad del capital estimado para los países en desarrollo (Banco Mundial, 2016; Gitman y Ramos, 2003).

\section{INGRESO NETO Y RENTABILIDAD}

Para ambas URP, el ingreso neto es positivo, en términos financieros y económicos; y los costos desembolsados indican que el productor obtiene un flujo de efectivo positivo (Tabla 4).

TABLA 4

Ingresos y costos de las URP analizadas en San Luis Potosí. (\$USD)

\begin{tabular}{|l|c|c|c|}
\hline Concepto & CD & CFIN & CEC \\
\hline Ingres os totales SLCC80 & $6.083,5$ & $6.083,5$ & $6.083,5$ \\
\hline Costos totales SLCC80 & $1.014,9$ & $1.413,1$ & $6.838,7$ \\
\hline Ingreso neto* SLCC80 & $5.068,6$ & $4.670,4$ & $-755,2-$ \\
\hline Ingres os totales SLCC100 & $8.020,9$ & $8.020,9$ & $8.020,9$ \\
\hline Costos totales SLCC100 & $1.166,8$ & $2.043,4$ & $8.651,2$ \\
\hline Ingreso neto* SLCC100 & 6.854 & $5.977,4$ & $-630,3$ \\
\hline Resultado & Con liquidez & Viable & Inviable \\
\hline
\end{tabular}

Del análisis realizado se concluye que ambas URP cuentan con liquidez y sus utilidades netas financieras son positivas, por lo que son viables en el corto y mediano plazo, no así en el largo plazo, dado que no alcanzan a cubrir el costo de oportunidad de los factores de producción; es decir, no están haciendo un uso eficiente de los recursos o factores que emplean en la producción.

Otra forma de analizar la rentabilidad es mediante la relación beneficio/costo; la cual para el caso de SLCC80 se estimó en 4,3 y para SLCC100 en 3,9 ambos cálculos se realizaron en términos financieros (Tabla 5). Estos resultados concuerdan con lo reportado en la literatura (Gómez, 2007; Cruz-Torres et al., 2011; Rebollar-Rebollar et al., 2012; Barrera-Perales, 2013; Orona Castillo et al., 2013; Orona et al., 2014), ya que parece haber un consenso general sobre la rentabilidad positiva de la ganadería caprina. De los resultados reportados por Sagarnaga-Villegas et al. (2015) se estimó una relación beneficio costo de 1,69 para un sistema estabulado de 10 vientres en el estado de Hidalgo, con los resultados reportados por Rebollar-Rebollar et al. (2012) se estimó una relación beneficio costo de 8,1, para un sistema extensivo de 65 vientres en el Estado de 
México; Orona et al. (2014), estimó para una granja de 100 vientres bajo un sistema semi-estabulado en el norte de México, una relación beneficio costo de 1,06; Barrera-Perales (2013) reportó una relación beneficio costo de 1,47 para un rebaño de más de 100 vientres en San Luis Potosí y Cruz-Torres et al. (2011) estimaron una relación beneficio costo de 1,8 para una granja de 35 vientres en pastoreo en Venezuela.

TABLA 5

Comparación del costo de producción y la ganancia en las URP caprinas de San Luis Potosí

\begin{tabular}{|l|c|c|c|c|}
\hline URP & Costos (USD/cab) & $\begin{array}{c}\text { Ingreso total } \\
\text { (USD/cab) }\end{array}$ & $\begin{array}{c}\text { Ganancia } \\
\text { (USD/cab) }\end{array}$ & Relación B/C \\
\hline SLCC80* & $17,7^{\star}$ & 76 & 46,7 & 4.3 \\
\hline SLCC80** & $85,5^{\star \star}$ & 76 & $-9,5$ & 0,9 \\
\hline SLCC100* & $20,4^{*}$ & 80,2 & 59,8 & 3,9 \\
\hline SLCC100** & $86,5^{\star \star}$ & 80,2 & $-6,3$ & 0,9 \\
\hline
\end{tabular}

Diversos autores coinciden en que la rentabilidad de este tipo de URP, en términos financieros, se debe principalmente al bajo costo de alimentación basado en pastoreo (Cruz-Torres et al., 2011; Rebollar-Rebollar et al., 2012; Orona et al., 2014).

Si la relación beneficio/costo se estima con base en los costos económicos, es de 0,9 para ambas URP; esto indica pérdidas económicas en las explotaciones, debido a que no se cubren los costos de los factores de producción, es decir, en términos económicos los costos son mayores a los beneficios.

\section{Precios de EQuilibrio}

Con base en la perspectiva de los panelistas se plantearon tres contextos de producción basados en los rendimientos de los principales productos generadores de ingreso y sus precios para obtener diferentes escenarios (Tabla 6) como lo propone Miragem et al. (1982). Los caprinocultores de ambas URP consensaron los rendimientos que podrían obtener bajo tres escenarios: optimista (buena disponibilidad de forraje), pesimista (sequía) y más probable (producción actual). Estos rendimientos coinciden con los estimados por Mellado, (2008) para cabras en agostaderos en el Norte de México.

TABLA 6

Rendimientos de queso y cabrito bajo diferentes escenarios en las URP analizadas en San Luis Potosí

\begin{tabular}{|l|l|c|c|c|}
\hline URP & Producto & Escenario más probable & $\begin{array}{c}\text { Escenario } \\
\text { optimista }\end{array}$ & $\begin{array}{c}\text { Escenario } \\
\text { pesimista }\end{array}$ \\
\hline SLCC80 & kg queso & 916 & 1,008 & 733 \\
\hline SLCC100 & kg queso & 1,143 & 1,258 & 915 \\
\hline SLCC80 & cabritos & 72 & 86 & 58 \\
\hline SLCC100 & cabritos & 110 & 132 & 88 \\
\hline
\end{tabular}


El precio de venta reportado por los panelistas fue de $\$ 4$ USD por kilogramo de queso, y de $\$ 25,4$ USD por cabrito, en ambas URP (Tabla 7). En el caso del queso, se observa que este precio permite tener un margen de ganancia, inclusive en el escenario pesimista de producción, sin embargo, el precio del cabrito presenta un margen menor, sobre todo bajo un escenario pesimista. De acuerdo con los panelistas, en el caso de oferta de animales, el precio llega a bajar hasta en 30\%, generando pérdidas estimadas en \$7,5 USD por cabrito.

TABLA 7

Precios de equilibrio bajo diferentes escenarios de producción (USD)

\begin{tabular}{|l|l|c|c|c|}
\hline URP & Unidades & $\begin{array}{c}\text { Escenario más } \\
\text { probable }\end{array}$ & $\begin{array}{c}\text { Escenario } \\
\text { optimista }\end{array}$ & $\begin{array}{c}\text { Escenario } \\
\text { pesimista }\end{array}$ \\
\hline SLCC80 & Queso $(\$ / \mathrm{kg})$ & 1,5 & 1,4 & 1,9 \\
\hline SLCC100 & Queso $(\$ / \mathrm{kg})$ & 1,8 & 1,6 & 2,2 \\
\hline SLCC80 & Cabritos $(\$ /$ cabeza) & 19,6 & 16,3 & 24,5 \\
\hline SLCC100 & Cabritos $(\$ /$ cabeza) & 18,6 & 15,5 & 23,2 \\
\hline
\end{tabular}

\section{Precios compuestos}

En la Tabla 8, se observa que ambas URP cubren CD y CFIN con la productividad de un vientre, sin embargo, para cubrir los CEC se requiere que se mejoren los parámetros productivos de los rebaños, es decir, para SLCC80 los rebaños deben mejorar su productividad en 13\% mientras que para SLCC100 esta debe incrementarse en $8 \%$. Como ya se mencionó, es posible alcanzar dichas mejoras en los rendimientos, sin embargo, debe considerarse un uso sustentable del agostadero.

TABLA 8

Costos de producción por vientre (USD/cabeza) y número de vientres necesarios para cubrirlos en las URP analizadas en San Luis Potosí

\begin{tabular}{|l|c|c|l|c|c|}
\hline \multicolumn{3}{|c|}{ SLCC80 } & \multicolumn{3}{c|}{ SLCC100 } \\
\hline Costos & $(\$ /$ vientre $)$ & Núm. vientres & Costos & (\$/vientre) & Núm. Vientres \\
\hline CD & 12.7 & 0.17 & CD & 11.7 & 0.15 \\
\hline CFIN & 17.7 & 0.23 & CFIN & 20.4 & 0.25 \\
\hline CEC & 85.5 & 1.13 & CEC & 86.5 & 1.08 \\
\hline
\end{tabular}

Los productores al cubrir los costos desembolsados y la depreciación de activos por la venta de queso y cabritos, generan un "excedente de producción” que de acuerdo a Wolf, (1971), es entendido como "la producción por encima del mínimo requerido para que los productores se mantengan", es decir, cuando los productores cubren los costos de depreciación de activos se habla entonces de "excedente de reemplazo", el cual es la cifra necesaria para reemplazar el equipo mínimo de producción y el cual se determina por aspectos técnicos y culturales de los productores. El excedente de reemplazo en la economía campesina, es el equivalente a los CFIN en el análisis de paneles de productores; ya que, dichos costos cubren la depreciación de los factores de producción. De acuerdo a Wolf (1971) “a partir de este nivel productivo, todo esfuerzo 
superior vendrá determinado por impulsos sociales y de mercado, en los que el campesinado está envuelto" es decir, buscará maximizar sus ganancias.

\section{FACTORES QUE INCIDEN EN LA RENTABILIDAD DE LA CAPRINOCULTURA}

De acuerdo con Wolf, (1971) y Schejtman, (1981); el uso de mano de obra exclusivamente familiar, el nivel tecnológico (manejo rústico del rebaño), la infraestructura limitada y la dependencia total del agostadero para alimentación del ganado, son los factores que explicarían la rentabilidad y continuidad de las explotaciones que operan bajo una lógica de producción campesina. Cabe destacar que en algunos estados (Texas, California y Florida) de la Unión Americana se está dando una reconversión productiva de la agricultura a ganadería caprina para carne, algunos de las razones que incentivan dicho cambio son el margen de rentabilidad y la facilidad con la cual todos los miembros de la familia se pueden integrar al manejo de la granja (Gillespie, Nyaupane, Dunn, \& McMillin, 2016). En otras unidades de producción agrícola en regiones (Zacatecas, México) similares al Altiplano Potosino se observaron como variables explicativas de la rentabilidad la mano de obra, el sistema de producción rustico y el usufructo de la tierra bajo el régimen de tenencia ejidal (Delgadillo-Ruiz, Valez-Cepeda, Ramírez-Moreno y Salas-González, 2016).

En el Altiplano Potosino, el agostadero es un recurso natural, cuyo costo de oportunidad está ligado a las actividades pecuarias, debido a que no tiene otro uso alternativo, es decir, son terrenos no aptos para la agricultura y otras actividades económicas, por lo que el aprovechamiento de éste por medio de la ganadería y la mano de obra familiar, permite generar ingreso como lo expresan Shanín, (1976) y Linck, (1992).

Se cree que, si el costo de oportunidad del pastoreo se asignara conforme al costo de alimentar al ganado en un sistema estabulado, los costos de alimentación se incrementarían considerablemente y con ello la rentabilidad de la actividad caprina se vería disminuida o dejaría de ser rentable.

La alimentación de los rebaños en el agostadero le confieren una ventaja comparativa (Parkin y Loria, 2010) a la ganadería extensiva con respecto a otros sistemas de producción mixtos o intensivos, sin embargo, al no asignar un costo al uso del agostadero y delimitar sus derechos de propiedad, se sobreexplota y se incurre en los problemas que genera el uso de los recursos comunes que aborda Garret Hardin (1968), en The tragedy of commons. ${ }^{(1)}$

En otras regiones de México, existe evidencia que indica que los rebaños caprinos tienden a crecer cuando su alimentación se basa en el pastoreo debido a su bajo costo, esto ocasiona que se acentúe la sobre carga y con ello el deterioro de los agostaderos (Martínez-González, Muñoz-Rodríguez, Santoyo-Cortés, Gómez-Pérez y Altamirano-Cárdenas, 2013).

Debe recordarse que, por lo general, los agostaderos no cuentan con alternativas productivas rentables diferentes a la ganadería (Negrete-Sánchez, Aguirre-Rivera, Pinos-Rodríguez y Reyes-Hernández, 2016); por lo que este costo tendría que ser estimado por la capacidad para producir leche y carne y luego por los servicios ambientales que ofrece (captura de carbono, captura de agua, resguardo de vida silvestre y paisaje) entre otros bienes y servicios ecosistémicos (Maczko et al., 2011).

\section{Conclusiones}

Se encontró que las URP analizadas cuentan con liquidez y son rentables, por lo que son financieramente viables en el corto y mediano plazo, ya que cubren necesidades de efectivo, depreciación de activos y costos de producción. La URP SLCC80 resultó ser la más rentable debido a los más altos rendimientos de queso y cabrito obtenidos.

La viabilidad de las URP puede mejorarse con el incremento en los rendimientos y la optimización de los parámetros productivos de los rebaños a través del manejo reproductivo y alimenticio, así como en la 
generación de valor agregado a la producción (elaboración de diferentes tipos de queso o y análisis de las cadenas de valor del queso y cabrito).

Bajo un enfoque de economía tradicional, se cuestionaría la permanencia de estas URP en el largo plazo; ya que los factores de producción tierra, mano de obra y capital no son remunerados. Sin embargo, el enfoque de economía campesina ayuda a entender por qué los productores permanecen en la actividad, y es precisamente el aprovechamiento de la mano de obra familiar y de los agostaderos; así como la posibilidad de obtener productos de autoconsumo a los cuales de otra manera no se tendría acceso. Lo anterior asegura la continuidad de esta actividad a pesar de no ser rentable en el largo plazo.

Se evidenció la capacidad de la ganadería caprina para generar ingreso y alimentos a partir de factores de producción (mano de obra y agostadero) que tienen usos alternativos limitados. Sin embargo, el no imputar un costo al uso de los agostadores distorsiona costos, rentabilidad y viabilidad de las URP, lo que repercute en sobrepastoreo de este recurso natural.

Cabe destacar que, en las zonas áridas, la ganadería extensiva depende exclusivamente del agostadero para su sustento, es por ello que cuantificar económicamente el valor de uso de este recurso natural, permitirá generar estrategias para su conservación.

Los análisis de costos, rentabilidad y viabilidad financiera y económica de la caprinocultura extensiva deben asignar un costo al uso de los agostaderos. De otra forma la ganadería extensiva seguirá presionando este recurso natural, poniendo en riesgo una de las pocas alternativas productivas disponibles en zonas áridas.

Este trabajo se centró en analizar la viabilidad económica de la ganadería caprina extensiva a través de una metodología que integrara conceptos de economía clásica y economía campesina que fueran equiparables con el objetivo de no dejar del lado elementos como la mano de obra de tipo familiar y el uso de agostaderos que en la mayoría de análisis económicos tradicionales no se cuantifican.

\section{Agradecimientos}

A la M.C. Betsaida Ángeles Hernández, facilitadora estatal de los Sistemas Producto Pecuarios de San Luis Potosí, por brindar las facilidades para la realización de los paneles y a todos los productores que participaron en este trabajo.

\section{REFERENCIAS}

Banco Mundial. (2016). Tasa de interés activa. Revisado en diciembre 22, 2016, de http://datos.bancomundial.org/ indicador/FR.INR.LEND?view $=$ chart

BANXICO, B. de M. (2017). Mercado cambiario en México. Revisado en marzo 4, 2017, de http://www.banxico.o rg.mx/portal-mercado-cambiario/

Barrera-Perales, O. T. (2013). Caracterización e importancia socioeconómica y ambiental de la producción caprina en el Altiplano Potosino. (Tesis de maestría), Universidad Autónoma de San Luis Potosí. San Luis Potosí, México.

Bartlett, E. T. O. M., Torell, L. A., Rimbey, N. R., Tassell, L. W. V. A. N., \& Daniel, W. (2002). Valuing grazing use on public land. Journal of Range Manage, 55 (September), 426-438.

Bartra, R. (1974). La teoría del valor y la economía campesina: invitación a la lectura de Chayanov.

Bartra, R. (1976). Introduccíón a Chayanov. Nueva Antropologia, I (3), 46-49.

Besley, S., y Bringham, F. (2008). Fundamentos de administración financiera (14a ed.). México, D. F.: Cengage Learning.

Castillo-Rodríguez, S., Rivera-Sandoval, J., González-Reyna, A., y Martinez-González, J. (2013). Comportamiento predestete de cabritos cruzados en Guanajuato, México. Revista MVZ Córdoba, 18, 3607-3611.

Cauderales, D. M., Milano, G. M. M. y Granda, Y. (2014). Caracterización Etnológica del ecotipo "Caprino Criollo" de la Microrregión Cauderales, Lara, Venezuela. Zootecnia Tropical, 32(3), 269-273. 
CEPAL Comisión Económica para América Latina y el Caribe. (2005). Pobreza, desertificación y degradación de los recursos naturales. (C. Morales y S. Parada, Eds.) (87th ed.). Santiago de Chile: Naciones Unidas-CEPAL.

CONAMI, C. N. de S. M. (2016). Salario mínimo en México por región. Revisado en marzo 8 de 2017 de: http://w ww.conasami.gob.mx/pdf/tabla_salarios_minimos/2016/01_01_2016.pdf

CONEVAL. (2017). Estadísticas de San Luis Potosí. Revisado en Marzo 8, 2017, de:http://www.cuentame.inegi.org $. \mathrm{mx} / \mathrm{monografias} /$ informacion $/ \mathrm{slp} /$ default.aspx?tema $=\mathrm{me} \& \mathrm{e}=24$

Coppock, D. L., Fernández-Giménez, M., Hiernaux, P., Huber-Sannwald, E., Schloeder, C., Valdivia, \& C.,Turner, M. (2017). Rangeland Systems in Developing Nations: Conceptual Advances and Societal Implications. In D. D. Briske (Ed.), Rangeland Systems: Processes, Management and Challenges (pp. 569-641). Cham: Springer International Publishing. http://doi.org/10.1007/978-3-319-46709-2_17.

Cruz-Torres, J. A., García-Hernández, L. A., Espinosa-Ortiz, V.E. y Araque-Herrera, C. A. (2011). Análisis económico del sistema de producción caprino en la parroquia de Montes de Oca, estado Lara, Venezuela. Revista Cientifica, 21(3), 239-245.

Delgadillo-Ruiz, O., Leos-Rodríguez, J. A., Valez-Cepeda, R. D., Ramírez-Moreno, P. P. y Salas-González, J. M. (2016). Análisis de la viabilidad de la producción de frijol (Phaseolus vulgaris L.) en el corto y largo plazo en Zacatecas, México. Agroproductividad, 9(5).

Domínguez-Torreiro, M., y Gómez-Rodríguez, F. (2013). Pagos agroambientales y productores agrarios: un análisis Delphi de las percepciones y demandas de los ganaderos beneficiarios de los programas de ayudas. Revista Española de Estudios Agrosociales y Pesqueros, 236, 81-118.

Dossa, L. H., Rischkowsky, B., Birner, R. \& Wollny, C. (2008). Socio-economic determinants of keeping goats and sheep by rural people in southern Benin. Agriculture and Human Values, 25(4), 581-592. http://doi.org/10.1 007/s10460-008-9138-9

Duflo, E., \& Udry, C. (2003). Intrahousehold resource allocation in Côte d'Ivoire: social norms, separate accounts and consumption choices. BREAD Working Paper No. 016. Bureau for Research and Economic Analysis.

Ensabella, B. (2008). El deterioro de los ecosistemas del norte cordobés y los límites de las economías campesinas. Mundo Agrario, 9(17).

FAO. (2017). Producción pecuaria en América Latina y el Caribe. Revisado en marzo 25, 2017, de: http://www.fao .org/americas/perspectivas/produccion-pecuaria/es/

Garcia, O. L. (1999). Administración financiera, fundamentos y aplicaciones (3a ed.). Cali, Colombia: Prensa Moderna Editores.

Gillespie, J., Nyaupane, N., Dunn, B., \& McMillin, K. (2016). Why do farmers decide to produce meat goats? Evidence from the United States. Agriculture and Human Values, 33(4), 911-927. http://doi.org/10.1007/ s10460-015-9677-9

Gitman, L. J., y Ramos, E. N. (2003). Principios de administración financiera. Pearson Educación.

Gómez, R. W.J. (2007). La caprinocultura como elemento articulador del desarrollo rural en el Altiplano Potosino. (Tesis doctoral), Universidad Autónoma de San Luis Potosí, San Luis Potosí, México.

Guzmán, C. M. G. (2014). Trashumancia relictual: la resistencia de las cabras. Estudios Sociales, XXIII (46), 343-346.

Hardin, G. (1968). The tragedy of commons. Science, 162, 1243-1248.

Hernández, H. E.J., Luna, C. L., Camacho, R. J. C., Franco, G. F.J., y Hernández, R. D. (2014). Producción y mercadeo de carne caprina en una región silvopastoril de la Mixteca Poblana, México. Revista Mexicana de Agronegocios, XVIII (35), 1043-1051.

Hof, J. G., McKean, J. R., Taylor, R. G., \& Bartlett, E. T. (1989). Contingent valuation of a quasi-market good: an exploratory case study offederal range forage RM-283. (U. F. S. R. Paper, Ed.) (Research p). Fort Collins, Colorado: USDA, Forest Service, Rocky Mountain Forest and Range Experiment Station.

INIFAP. (2008). Coeficientes de agostadero para San Luis Potosí. Revisado en abril 26 de 2017 de: http://biblioteca .inifap.gob.mx:8080/xmlui/bitstream/handle/123456789/1237/472.pdf?sequence=1

Landini, F. (2011). Racionalidad económica campesina. Mundo Agrario, 12(23). 
Linck, T. (1992). Cambio técnico y marco macroeconómico de la "modernización" de la agricultura campesina. Revisado en agosto 25 de 2017, de: http://www.colmich.edu.mx/relaciones25/files/revistas/049/ThierryLinc k.pdf

López, G. J. L., Damián, H. M. A., Álvarez, G. F., Parra, I. F. y Zuluaga, S. G. P. (2012). La economía de traspatio como estrategia de supervivencia en San Nicolás de los Ranchos, Puebla, México. Revista de Geografía Agrícola, $48-49,51-62$.

Maczko, K., Tanaka, J. a, Breckenridge, R., Hidinger, L., Heintz, H. T., Fox, W. E., McCollum, D. W. (2011). Rangeland Ecosystem Goods and Services: Values and Evaluation of Opportunities for Ranchers and Land Managers. Rangelands, 33, 30-36. http://doi.org/10.2111/1551-501X-33.5.30

Martínez-González, E. G., Muñoz-Rodríguez, M., Santoyo-Cortés, V.H., Gómez-Pérez, D., \& Altamirano-Cárdenas, J. R. (2013). Lecciones de la promoción de proyectos caprinos a través del programa estratégico de seguridad alimentaria en Guerrero, México. Agricultura, Sociedad y Desarrollo, 10(2), 177-193.

Mellado, M. (2008). Técnicas para el manejo reproductivo de las cabras en agostadero. Tropical and Subtropical Agroecosystems, 9, 47-63.

Miragem, S., Pietra, E., Fuentes, N., Nadal, F., Porteiro, J., Sánchez, B., \& Vázquez, R. (1982). Guia para la elaboración de proyectos de desarrollo agropecuario (IICA). San José, Costa Rica.

Miranda-Aragón, L., Treviño-Garza, E. J., González-Tagle, M. A., Pompa-Garcia, M. y Aguirre-Salado, C. A. (2011). Tasa de deforestación en San Luis Potosí, México (1993-2007). Revista Chapingo Serie Ciencias Forestales y Del Ambiente, 44(201-215), 15. http://doi.org/10.5154/r.rchscfa.2011.06.044

Negrete-Sánchez, L. O., Aguirre-Rivera, J. R., Pinos-Rodríguez, J. M., \& Reyes-Hernández, H. (2016). Beneficio de la parcelación de los agostaderos comunales del ejido "El Castañón", municipio Catorce, San Luis Potosí: 1993-2013. Agrociencia, 50, 511-532.

Orona, C. I., Fortis, H. M., Gallegos, R. M. Á., Salazar, S. E., \& García, H. J. L. (2014). Proyección económica de Unidades Representativas de Producción Caprina en la Comarca Lagunera, México. Revista Mexicana de Agronegocios, XVIII (35), 1111-1120.

Orona Castillo, I., Sangerman-Jarquín, D. M., Antonio-González, J., Salazar Sosa, E., García Hernández, J. L., NavarroBravo, A., y Schwentesius de Rindermann, R. (2013). Proyección económica de unidades representativas de producción en caprinos en la Comarca Lagunera, México. Revista Mexicana de Ciencias Agrícolas, 4, 625-636.

Orozco Hernández, M. E., y López Andrés, D. (2007). Estrategia de supervivencia familiar en una comunidad campesina del Estado de México. Ciencia Ergo Sum, 14(3), 246-254.

Parkin, M., y Loria, D. E. (2010). Microeconomia. Versión para Latinoamérica (9th ed.). México, D.F.: Pearson Educación.

Pearson, S., Gotsch, C., \& Sjaiful, B. (2004). Applications of the Policy Analysis Matrix in Indonesian Agriculture. Yayasan Obor Indonesia.

Pesántez, M. T., y Hernández, A. (2014). Producción lechera de cabras Criollas y Anglo-Nubian en Loja, Ecuador. Revista Cubana de Ciencia Agrícola, 48(2), 105-108.

Pimienta, L. R. (2000). Encuestas probabilísticas vs. no probabilísticas. Política Y Cultura, 13, 263-276. Extraído de: http://www.redalyc.org/articulo.oa?id=26701313

Rebollar-Rebollar, S., Hernández-Martínez, J., Rojo-Rubio, R., y Guzmán-Soria, E. (2012). Gastos e ingresos en la actividad caprina extensiva en México. Agronomia Mesoamericana, 23(1), 159-165.

Sagarnaga-Villegas, L. M., y Salas-González, J. M. (2010). Unidades Representativas de Producción Pecuaria: Panoráma Económico 2008-2018.

Sagarnaga-Villegas, L. M., Salas-González, J. M., y Aguilar-Avila, J. (2015). Production costs, equilibrium and target prices of a representative goat farm in Hidalgo, México. In XIV International Business and Economy Conference (pp. 1-14). Bangkok, Thailand.

Sagarnaga-Villegas, L. M., Salas-González, J. M. y Aguilar-Ávila, J. (2014). Ingresos y Costos de Producción 2013. Unidades Representativas de Producción. Trópico Húmedo 2013. Paneles de Productores. (S. y T. de la A. y la A. 
M. (CIESTAAM) Centro de Investigaciones Económicas, Ed.) (Primera ed). México: Universidad Autónoma Chapingo/CIESTAAM.

Sagarnaga-Villegas, L. M., Salas-González, J. M., Aguilar-Avila, J., Gómez-González, G. y Suarez-Dominguez, H. (2014). Costos de producción caprina, precios de equilibrio y objetivo de una URP caprina en Hidalgo, México. Paneles de productores. In Ingresos y costos de producción 2013 (CIESTAAM, pp. 731-743). Universidad Autónoma Chapingo/CIESTAAM.

Salas G., J., Sagarnaga, V. M., Gómez, G. G., Leos, R. J. y Peña, J. S. (2013). Unidades Representativas de Producción de Cereales. Panorama Económico 2009-2014. Estado De Guanajuato. Revista Mexicana de Administración Agropecuaria A.C., XIII(33), 483-494.

Santacoloma- Varón, L. E. (2015). Importancia de la economía campesina en los contextos contemporáneos: una mirada al caso colombiano. Entramado, 11(2), 38-50.

Santarcángelo, J. E., y Fal, J. (2009). Producción y rentabilidad en la ganadería argentina. 1980-2006. Mundo Agrario, 10(19).

Schejtman, A. Z. (1981). Economía campesina y agricultura empresarial: Tipología de productores del agro mexicano (1a ed.). Comisión Económica para América Latina (CEPAL).

Schejtman, A. Z. (1995). Elementos para una teoría de la economía campesina: pequeños propietarios y campesinos de hacienda. El Trimestre Económico, 42(166(2)), 487-508.

SENASICA. (2017). Campaña Nacional Contra la Bruecelosis del Ganado. Revisado en agosto 25 de 2017, de: https ://www.gob.mx/senasica/documentos/situacion-actual-del-control-de-la-brucelosis-en-mexico

Shanín, T. (1976). Naturaleza y lógica de la economía campesina. Anagrama (Vol. 1).

SIAP, y SAGARPA. (2015). Centro de Estadística Agropecuaria. Sistema de Información Agropecuaria de Consulta 1980-2013. (SIACON). Versión 38.0. México, D.F.

Silvestre, P., Naim, P., Cueto, M., y Gibbons, A. (2012). Estacionalidad reproductiva en machos caprinos criolloneuquinos de la Patagonia Argentina. Archivos de Zootecnia, 61(233), 119-128.

Suárez, V. H., Fondraz, M., Viñabal, A. E., Martínez, G. M., y Salatin, A. O. (2013). Epidemiología de los nematodos gastrointestinales en caprinos lecheros en los valles templados del NOA, Argentina. RIA, 29(2), 191-197.

Torres-Vázquez, J. A., Valencia-Posadas, M., Castillo-Juárez, H., y Montaldo, H. H. (2010). Tendencias genéticas y fenotípicas para características de producción y composición de la leche en cabras Saanen de México. Revista Mexicana de Ciencias Pecuarias, 1(4), 337-348.

Torres Rivera, J. A., Mellado Bosque, M., Beltrán López, S., Ortega Reyes, L., Echavarría Cháirez, F. G., López García, J. C. y Ramírez Lozano, R. G. (2013). Tecnologias en apoyo a la caprinocultura (Vol. I).

UNAM, (Universidad Nacional Autónoma de México). (2012). Sistema de Costos, Eficiencia y Competitividad de los Sistemas Pecuarios en México. Informe del sistema caprino. Encuesta 2012. (Universidad Nacional Autónoma de México. Facultad de Medicina Veterinaria y Zootecnia., Ed.). México, D.F.: Secretaría de Agricultura, Ganadería, Desarrollo Rural, Pesca y Alimentación. Dirección General de Ganadería.

United States Department of Agriculture-Economic Research Service, (USDA-ERS). (2012). Commodity costs and returns. Economic Research Service.

United States Department of Agriculture - Natural Resources Conservation Service, (USDA-NRCS). (2000). Commodity costs and returns Estimation Handbook. A Report of the AAEA Task Force on Commodity Costs and Returns. (A. R. of the A. T. F. on C. C. and Returns, Ed.). Iowa, EEUU.

Vargas-Canales, J. M., Palacios-Rangel, M. I., Acevedo-Peralta, A. I. y Leos-Rodríguez, J. A. (2015). Profitability analysis for natural rubber (Hevea brasiliensis Muell. Arg.) production in Oaxaca, Mexico. Revista Chapingo, Serie Ciencias Forestales y Del Ambiente, 22(1), 45-58. http://doi.org/10.5154/r.rchscfa.2015.02.005

Wolf, E. R. (1971). Los campesinos. Barcelona, España: Editorial Labor.

Zavala, M.J., Salas, G. J., Leos, R. J. y Sagarnaga, V. M. (2012). Construcción de unidades representativas de producción porcina y análisis de su viabilidad económica en el período 2009-2018. Agrociencia, 46(7). 


\section{Notas}

(1) En este trabajo, Hardin ejemplifica con un pastizal natural de acceso abierto, la problemática que presentan los recursos de uso común en ausencia de reglas para su aprovechamiento. Este, autor evidencia el deterioro ocasionado por el sobrepastoreo oportunista que los ganaderos hacen al incrementar el tamaño de sus hatos en el pastizal hasta agotar este recurso. A pesar del conocimiento que tienen los usuarios sobre las consecuencias de sus decisiones individuales de tener hatos cada vez más grandes ninguno de ellos dejará de adicionar más animales ya que nada les garantiza que su compañero lo haga, sino por el contrario tienen la certeza que éste al igual que él lo siga incrementado. Se concluye que los recursos de uso común deben ser privatizados para que los ganaderos manejen el pastizal como un capital que tiene que preservarse, o se definen normas colectivas para reglamentar el acceso a los recursos comunes y asegurar su conservación. 